\title{
The role of postoperative radiotherapy in stage II and III thymoma: a Korean multicenter database study
}

\author{
Seung Hwan Song ${ }^{1}$, Jee Won Suh ${ }^{2}$, Woo Sik Yu ${ }^{3}$, Go Eun Byun ${ }^{4}$, Seong Yong Park ${ }^{4}$, Chang Young Lee ${ }^{4}$, \\ Dae Joon Kim ${ }^{4}$, Hyo Chae Paik ${ }^{4}$, Kyung Young Chung ${ }^{4}$, Geun Dong Lee ${ }^{5}$, Sehoon Choi ${ }^{5}$, \\ Hyeong Ryul Kim ${ }^{5}$, Yong-Hee Kim ${ }^{5}$, Dong Kwan Kim ${ }^{5}$, Seung-Il Park ${ }^{5}$, Jong Ho Cho ${ }^{6}$, \\ Hong Kwan Kim ${ }^{6}$, Yong Soo Choi ${ }^{6}$, Jhingook Kim ${ }^{6}$, Jae Il Zo ${ }^{6}$, Young Mog Shim ${ }^{6}$, Yoohwa Hwang ${ }^{7}$, \\ Samina Park ${ }^{8}$, In Kyu Park ${ }^{8}$, Chang Hyun Kang ${ }^{8}$, Young Tae Kim ${ }^{8}$, Jin Gu Lee
}

${ }^{1}$ Department of Thoracic and Cardiovascular Surgery, Gangnam Severance Hospital, Yonsei University College of Medicine, Seoul, Republic of Korea; ${ }^{2}$ Department of Thoracic and Cardiovascular Surgery, Yongin Severance Hospital, Yonsei University College of Medicine, Yongin, Republic of Korea; ${ }^{3}$ Department of Thoracic and Cardiovascular Surgery, Ajou University School of Medicine, Suwon, Republic of Korea; ${ }^{4}$ Department of Thoracic and Cardiovascular Surgery, Severance Hospital, Yonsei University College of Medicine, Seoul, Republic of Korea; ${ }^{5}$ Department of Thoracic and Cardiovascular Surgery, Asan Medical Center, Ulsan University College of Medicine, Seoul, Republic of Korea; ${ }^{6}$ Department of Thoracic and Cardiovascular Surgery, Samsung Medical Center, Sungkyunkwan University School of Medicine, Seoul, Republic of Korea; ${ }^{7}$ Department of Thoracic and Cardiovascular Surgery, Seoul National University Bundang Hospital, Seoul National University College of Medicine, Seongnam, Republic of Korea; ${ }^{8}$ Department of Thoracic and Cardiovascular Surgery, Seoul National University Hospital, Seoul National University College of Medicine, Seoul, Republic of Korea

Contributions: (I) Conception and design: SH Song, JG Lee; (II) Administrative support: GE Byun; (III) Provision of study materials or patients: All authors; (IV) Collection and assembly of data: SH Song; (V) Data analysis and interpretation: SH Song, JG Lee; (VI) Manuscript writing: All authors; (VII) Final approval of manuscript: All authors.

Correspondence to: Jin Gu Lee. Department of Thoracic and Cardiovascular Surgery, Severance Hospital, Yonsei University College of Medicine, 50-1 Yonsei-ro, Seodamun-gu, Seoul 03722, Republic of Korea. Email: csiglee@yuhs.ac.

Background: Complete resection is a standard treatment for patients with Masaoka-Koga stages II and III thymoma, however the role of postoperative radiotherapy (PORT) is controversial. We analyzed data collected from 4 Korean hospitals to determine the effectiveness of PORT in stage II and III thymoma patients.

Methods: Between January 2000 and December 2013, 1,663 patients underwent surgery for thymic tumors at the 4 hospitals. Among them, 668 patients (527 with stage II and 141 with stage III) were investigated, among whom, 443 received PORT (335 with stage II and 108 with stage III). Propensity score matching (PSM) was performed, and 404 patients (346 with stage II and 58 with stage III) were selected.

Results: Perioperative characteristics were similar in the PORT and non-PORT groups after PSM. On survival analysis of stage II patients, the PORT and non-PORT groups showed no difference in either 5-year recurrence-free survival (RFS) $(96.3 \%$ vs. $96.6 \%, \mathrm{P}=0.622)$ or 5 -year overall survival (OS) $(94.6 \%$ vs. $93.8 \%$, $\mathrm{P}=0.839)$. However, among stage III patients, the PORT group showed significantly better 5 -year RFS (75.7\% vs. $50.1 \%, \mathrm{P}=0.040)$ and 5 -year $\mathrm{OS}(86.5 \%$ vs. $54.7 \%, \mathrm{P}=0.001)$. On multivariate Cox regression analysis, PORT was a significant positive prognostic factor in terms of both RFS $(\mathrm{P}=0.005)$ and OS $(\mathrm{P}=0.004)$ in patients with stage III thymomas, but not in those with stage II disease ( $\mathrm{P}=0.987$ and 0.968 , respectively).

Conclusions: PORT improved the RFS and OS in stage III thymoma patients, but showed no survival benefit in stage II patients.

Keywords: Thymoma; postoperative radiotherapy (PORT); stage II; stage III

Submitted Apr 20, 2020. Accepted for publication Sep 17, 2020.

doi: $10.21037 /$ jtd-20-1713

View this article at: http://dx.doi.org/10.21037/jtd-20-1713 


\section{Introduction}

Thymomas are rare tumors that originate from thymic epithelial cells. While they have a relatively low incidence rate (accounting for only $0.2-1.5 \%$ of all malignancies) (1), thymomas are the most common mediastinal mass in adults, comprising $20-30 \%$ of all such masses (2). Because tumor-node-metastasis (TNM) staging is not useful for thymomas, the World Health Organization (WHO) histologic subtypes (3) or the Masaoka classification (4) are widely used for staging thymic epithelial tumors.

The Masaoka staging system has previously been shown to be a good predictor of prognosis and survival in patients with thymomas $(5,6)$; moreover, the International Thymic Malignancy Interest Group (ITMIG) introduced a modified Masaoka system for staging thymoma (Koga et al.) (7). The treatment of thymomas may involve surgical resection, chemotherapy, radiation therapy (RT), or combined therapies that are determined by disease stage. Of the multimodal therapies available, complete surgical resection remains the mainstay of treatment for thymomas $(5,6)$. RT has also been used as an adjuvant or palliative treatment since thymomas are radiosensitive (8); however, the effectiveness of postoperative radiotherapy (PORT) remains unclear. According to the ITMIG definitions and reporting guidelines for thymic malignancies (9), PORT is not indicated following complete resection of a wellencapsulated thymoma but should be considered in rare cases in which a noninvasive thymoma is not completely resected or when a patient is a poor surgical risk. In this study, we researched the database of the Korean Association for Research on the Thymus (KART) to determine the efficacy of PORT in patients with thymomas, especially those with Masaoka-Koga (M-K) stages II and III disease.

We present the following article in accordance with the STROBE reporting checklist (available at http://dx.doi. org/10.21037/jtd-20-1713).

\section{Methods}

\section{Data sources and patient selection}

The KART is a research organization established for the development and understanding of thymic epithelial tumors. The database comprises the electronic medical records of 1,663 patients collected from 4 Korean institutions between January 2000 and December 2013. For our study, patients who had benign diseases, M-K stage I and IV thymomas, thymic carcinomas, or neuroendocrine carcinomas were excluded, as were patients with missing data. Propensity score matching (PSM; 1:1) was performed to adjust for age, sex, myasthenia gravis, adjuvant chemotherapy, extent of surgery, postoperative complications, M-K stage, WHO subtypes, pathologic tumor size, complete resection, and postoperative chemotherapy (POCT). The WHO subtypes were divided into 2 groups based on prognostic relevance and statistical effectiveness: patients with $\mathrm{A}, \mathrm{AB}$, and $\mathrm{B} 1$ subtypes and those with B2 and B3 subtypes. After PSM, the PORT and non-PORT groups each comprised 202 patients. Both groups were analyzed retrospectively. The study was conducted in accordance with the Declaration of Helsinki (as revised in 2013). The study was approved by institutional review board (No. 4-2020-0861) and informed consent was taken from all individual participants.

\section{Definitions}

In this study, overall survival (OS) was defined as the length of time from the date of the surgical resection to the last follow-up date. And, recurrence-free survival (RFS) was defined as the time from the surgery to the last follow-up date without recurrence.

\section{Statistical analysis}

The baseline characteristics of each group were analyzed using the independent $\mathrm{t}$-test for continuous variables and the chi-square and Fisher's exact test for categorical variables. The paired $t$-test and McNemar's test were used for continuous and categorical variables, respectively, after performing PSM to adjust for confounding variables. Continuous data are expressed as means with standard deviations or as medians and ranges, whereas categorical variables are shown as counts and percentages. OS and recurrence-free survival were calculated from the date of resection and were estimated using the Kaplan-Meier method; differences were evaluated using the log-rank test. The Cox proportional hazards model was used to evaluate the influence of PORT and other risk factors on RFS and OS. Clinicopathological factors were identified using univariable analysis, and all factors with $\mathrm{P}$ values $<0.2$ were subjected to multivariable Cox regression analyses. Results are expressed as hazard ratios (HRs) and their corresponding $95 \%$ confidence intervals. All $\mathrm{P}$ values were 2 -sided, and a $\mathrm{P}$ value $<0.05$ was considered significant. All statistical analysis was performed using SPSS 23.0 (IBM, Armonk, NY, USA). 


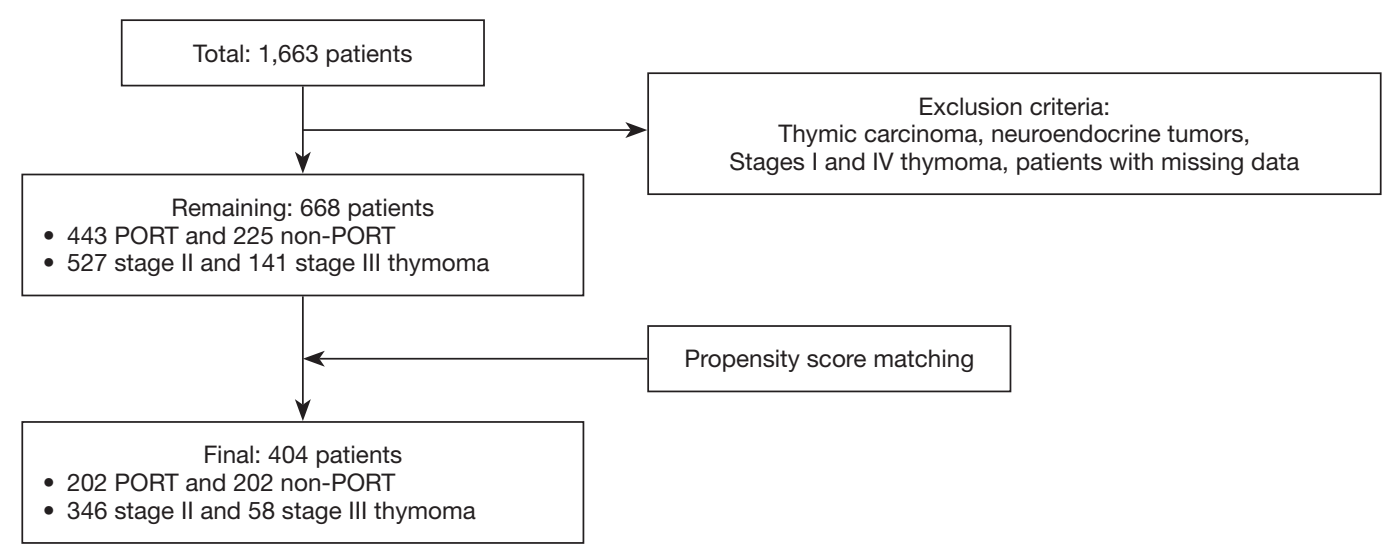

Figure 1 Scheme of the selection of the study population. PORT, postoperative radiotherapy.

\section{Results}

\section{Baseline characteristics}

Figure 1 shows the patient selection scheme. Of the 668 patients who met our inclusion criteria (527 with stage II and 141 with stage III thymomas), 443 (66.3\%) underwent PORT (of whom 335 had stage II and 108 had stage III disease). The median ages in the non-PORT and PORT groups were 53.0 and 50.0 years, respectively, representing a significant difference $(\mathrm{P}=0.031)$. Myasthenia gravis was more common in the PORT group ( $30.7 \%$ vs. $21.3 \%, \mathrm{P}=0.013)$; the $\mathrm{A} / \mathrm{AB} / \mathrm{B} 1$ subtypes were more commonly found in the non-PORT group (61.3\% vs. $32.1 \%)$, while B2/B 3 subtypes were more frequent in the PORT group $(67.9 \%$ vs. $38.7 \%$; $\mathrm{P}<0.001)$. Patients with $\mathrm{M}-\mathrm{K}$ stage II were more common than those with stage III in both groups; however, the proportion of stage II patients was higher in the nonPORT group ( $85.3 \%$ vs. $75.6 \%, \mathrm{P}=0.005)$. There were no differences between the groups in term of sex, adjuvant chemotherapy, extent of surgical resection, pathologically confirmed tumor size, rate of complete resection, and postoperative POCT (Table 1).

Following PSM, 404 patients were selected including 346 with stage II and 58 with stage III (Table 1); the matched groups were well-balanced. There were no significantly different factors between the non-PORT and PORT groups, including among those that were significantly different pre-PSM (i.e., myasthenia gravis, WHO subtypes, and M-K stage). The median age was 51.5 years in the nonPORT group and 50.0 years in the PORT group $(\mathrm{P}=0.420)$.

Subgroup analysis according to $\mathrm{M}-\mathrm{K}$ stage revealed that 172 stage II patients and 30 stage III patients received PORT; none of the investigated factors were significantly different between the non-PORT and PORT groups among patients with either disease stage.

\section{RFS and $O S$}

The 5-year RFS rates were not significantly different between the non-PORT and PORT patients overall (91.1\% vs. $93.0 \%, \mathrm{P}=0.373)$, or between those with stage II disease (96.6\% vs. 96.3\%, $\mathrm{P}=0.622$ ). However, among stage III patients, the 5-year RFS rate was significantly better in the PORT group than in the non-port group $(75.7 \%$ vs. $50.1 \%$, $\mathrm{P}=0.040$ ) (Figure 2).

Furthermore, OS was significantly longer in stage III patients receiving PORT (Figure 2). The 5- and 10-year OS rates were both $86.5 \%$ in the PORT group, but were $54.7 \%$ and $30.4 \%$ in the non-PORT group, respectively $(\mathrm{P}=0.001)$. However, there was no significant differences among stage II patients, where the 5- and 10-year OS rates were $94.6 \%$ and $84.3 \%$ in the PORT group, and $93.8 \%$ and $87.5 \%$ in the non-PORT group, respectively $(\mathrm{P}=0.839)$. Among all patients, the OS of those receiving PORT was not superior to that of patients in the non-PORT group. The 5 - and 10 -year OS rates were $93.1 \%$ and $85.1 \%$ in the PORT group, and $87.5 \%$ and $77.9 \%$ in the non-PORT group, respectively $(\mathrm{P}=0.160)$. Among stage III thymoma, three patients without PORT died within the postoperative 1 year. The cause of deaths were 1 myocardial infarction, 1 thymoma related death, and 1 unknown cause.

\section{Multivariate analysis of RFS}

On univariate analysis of all patients (Table 2), poorer RFS was associated with neoadjuvant chemotherapy, B2/ 
Table 1 Baseline characteristics of patients with thymoma before and after propensity score matching

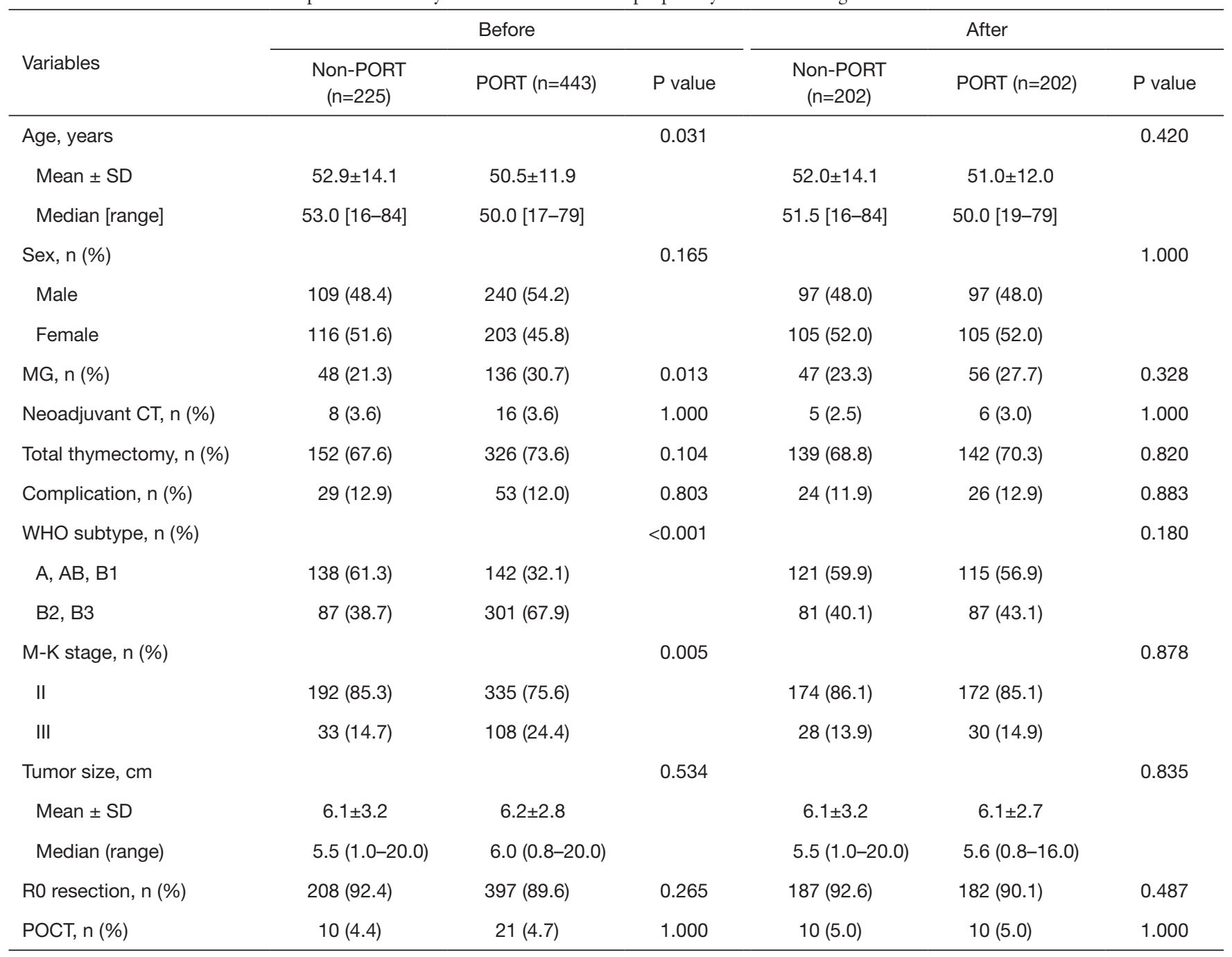

PORT, postoperative radiotherapy; SD, standard deviation; MG, myasthenia gravis; CT, chemotherapy; WHO, World Health Organization; $\mathrm{M}-\mathrm{K}$, Masaoka-Koga; R0 resection, complete resection; POCT, postoperative chemotherapy.

B3 WHO subtype, M-K stage III, pathologic tumor size, and POCT. In contrast, complete resection was associated with improved RFS. On multivariate analysis, only WHO subtypes B2/B3 (HR, 2.82; 95\% confidence interval (CI), 1.23-6.45; $\mathrm{P}=0.014)$ and $\mathrm{M}-\mathrm{K}$ stage III (HR, 5.22; 95\% CI, 2.48-10.97; $\mathrm{P}<0.001)$ showed higher HRs. PORT did not produce a significant change in the HR according to either univariate or multivariate analysis (HR, 0.57; 95\% CI, $0.28-$ $1.17 ; \mathrm{P}=0.123$ ).

In stage II patients (Table 3), complete resection was associated with improved RFS (HR, 0.09; 95\% CI, 0.02$0.43 ; \mathrm{P}=0.002)$. In contrast, neoadjuvant chemotherapy was associated with a higher HR (45.84; 95\% CI, 4.01-524.19;
$\mathrm{P}=0.002)$. PORT did not produce a significant different in HR among patients with stage II thymoma (HR, 1.01; 95\% CI, 0.31-3.33; $\mathrm{P}=0.987)$.

In stage III patients (Table 3), only PORT was associated with improved RFS on both univariate analysis (HR, 0.35; 95\% CI, 0.12-1.00; $\mathrm{P}=0.049$ ) and multivariate analysis (HR, $0.10 ; 95 \%$ CI, 0.02-0.50; $\mathrm{P}=0.005$ ).

\section{Multivariate analysis of $O S$}

Among all patients, old age (HR, 1.05; 95\% CI, 1.02-1.08; $\mathrm{P}=0.001$ ), neoadjuvant chemotherapy (HR, 2.87; 95\% CI, 1.01-8.12; $\mathrm{P}=0.047$ ), postoperative complications (HR, 4.01; 

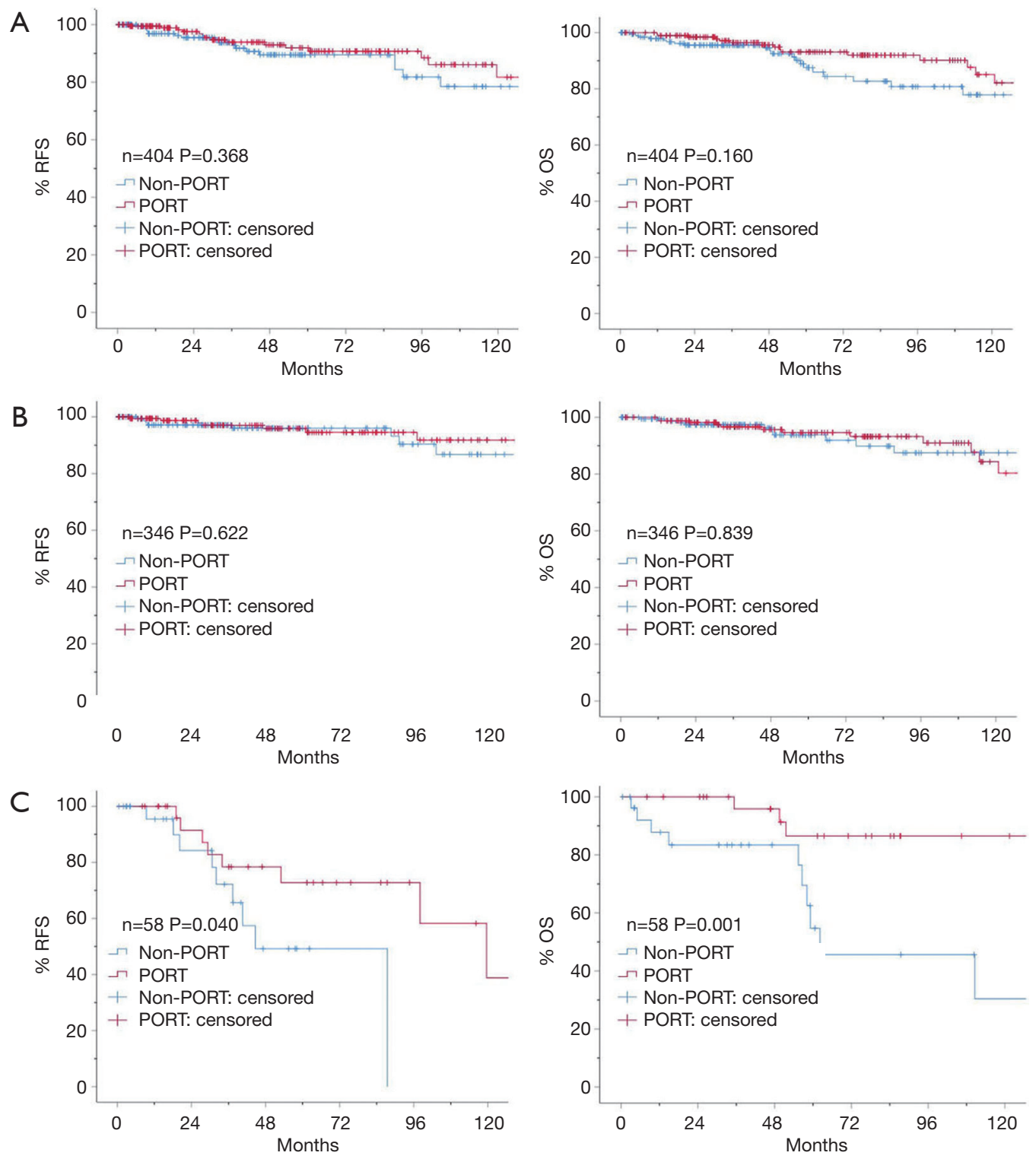

Figure 2 Kaplan-Meier curves and log-rank P values of recurrence-free and overall survival in the PORT versus non-PORT groups. (A) Stages II and III thymoma; (B) stage II thymoma; (C) stage III thymoma. PORT, postoperative radiotherapy; RFS, recurrence-free survival; OS, overall survival

95\% CI, 1.96-8.21; $\mathrm{P}<0.001)$, and recurrence (HR, 4.11; 95\% CI, 1.76-9.62; $\mathrm{P}=0.001)$ were associated with poor OS. In contrast, female sex (HR, 0.47; 95\% CI, 0.23-0.97; $\mathrm{P}=0.040$ ) was associated with longer OS (Table 2). There was no significant different in OS based on administering PORT (HR, 0.68; 95\% CI, 0.35-1.33; P=0.259).

In stage II patients (Table 4), older age (HR, 1.06; 95\% CI, 1.03-1.11; $\mathrm{P}=0.001)$, postoperative complications (HR, 5.48; 95\% CI, 2.22-13.52; $\mathrm{P}<0.001)$, WHO subtypes B2/B3
(HR, 3.82; 95\% CI, 1.33-10.95; $\mathrm{P}=0.013)$, and recurrence (HR, 10.43; 95\% CI, 3.27-33.32; $\mathrm{P}<0.001$ ) were associated with poorer OS. Female sex was associated with a more favorable OS in stage II patients (HR, 0.30; 95\% CI, 0.12 0.77; $\mathrm{P}=0.013)$. However, PORT was not associated with improved OS (HR, 0.98; 95\% CI, 0.40-2.43; P=0.968).

As with RFS, PORT was the only factor that was significantly associated with improved OS in patients with stage III thymomas (HR, 0.17; 95\% CI, 0.05-0.56; 
Table 2 Univariate and multivariate analysis of recurrence-free survival and overall survival in all patients (total $\mathrm{n}=404$ )

\begin{tabular}{|c|c|c|c|c|c|c|c|c|}
\hline \multirow{2}{*}{ Variables } & \multicolumn{4}{|c|}{ RFS } & \multicolumn{4}{|c|}{ OS } \\
\hline & $\mathrm{HR}(95 \% \mathrm{Cl})$ & $P$ value & $\mathrm{HR}(95 \% \mathrm{Cl})$ & $P$ value & HR (95\% Cl) & $P$ value & HR (95\% Cl) & $P$ value \\
\hline Age & $0.98(0.95-1.01)$ & 0.163 & $0.98(0.95-1.00)$ & 0.105 & 1.05 (1.02-1.07) & 0.001 & 1.05 (1.02-1.08) & 0.001 \\
\hline Sex & & 0.105 & & 0.228 & & 0.003 & & 0.040 \\
\hline Female & $0.56(0.27-1.13)$ & & $0.64(0.31-1.33)$ & & $0.36(0.18-0.71)$ & & $0.47(0.23-0.97)$ & \\
\hline$M G$ & $1.37(0.66-2.84)$ & 0.406 & $1.23(0.59-2.57)$ & 0.576 & $0.93(0.47-1.84)$ & 0.831 & $1.53(0.67-3.46)$ & 0.313 \\
\hline Neoadjuvant CT & $8.68(3.28-23.00)$ & $<0.001$ & $1.67(0.57-4.92)$ & 0.355 & $6.30(2.43-16.44)$ & $<0.001$ & $2.87(1.01-8.12)$ & 0.047 \\
\hline Total thymectomy & $0.45(0.17-1.15)$ & 0.096 & $0.54(0.22-1.32)$ & 0.177 & $0.80(0.41-1.59)$ & 0.53 & $0.65(0.31-1.35)$ & 0.244 \\
\hline$A, A B, B 1$ & Reference & & Reference & & Reference & & Reference & \\
\hline B2, B3 & $4.20(1.88-9.36)$ & & $2.82(1.23-6.45)$ & & $1.12(0.60-2.09)$ & & $2.28(1.01-5.18)$ & \\
\hline M-K stage & & $<0.001$ & & $<0.001$ & & $<0.001$ & & 0.313 \\
\hline II & Reference & & Reference & & Reference & & Reference & \\
\hline III & $8.24(4.09-16.61)$ & & $5.22(2.48-10.97)$ & & $3.36(1.77-6.37)$ & & $1.56(0.66-3.66)$ & \\
\hline Pathologic size & $1.12(1.02-1.23)$ & 0.022 & $1.01(0.90-1.13)$ & 0.882 & $1.04(0.94-1.15)$ & 0.432 & $1.05(0.95-1.17)$ & 0.339 \\
\hline R0 resection & $0.27(0.13-0.59)$ & 0.001 & $0.45(0.201 .00)$ & 0.051 & $1.00(0.38-2.60)$ & 0.994 & $1.68(0.57-4.96)$ & 0.352 \\
\hline POCT & 4.05 (1.75-9.38) & 0.001 & $1.83(0.77-4.37)$ & 0.174 & $2.15(0.89-5.21)$ & 0.089 & $3.61(1.26-10.31)$ & 0.017 \\
\hline
\end{tabular}

HR, hazard ratio; Cl, confidence interval; MG, myasthenia gravis; CT, chemotherapy; WHO, World Health Organization; M-K, MasaokaKoga; R0 resection, complete resection; POCT, postoperative chemotherapy; PORT, postoperative radiotherapy.

$\mathrm{P}=0.004)($ Table 4).

\section{Discussion}

The choice of treatment is the most important decision physicians make regarding their patients. However, thymic epithelial tumors are rare, and the heterogeneity of treatment recommendations as well as the variations in reported outcomes can cause uncertainty regarding the optimal treatment for this disease. PORT is conventionally administered to patients with thymomas in clinical practice; however, its effectiveness has remained unclear (10-12), and there is no established consensus on its indication $(13,14)$. M-K stage II and III thymomas are both locally invasive but non-metastatic; however, stage II thymoma is considered an early-stage tumor that has a low recurrence rate, whereas stage III thymoma is an advanced-stage tumor with a relatively high rate of recurrence (15). Many studies have evaluated the efficacy of PORT for the treatment of thymomas; however, their results were inconsistent. Some researchers found that the benefit of PORT in patients with stage II thymoma is questionable, whereas its efficacy in patients with stage III has not yet been established (10-12,16-25).

Omasa $\mathrm{et} \mathrm{al}$. investigated the effectiveness of PORT in 1,265 patients with stage II and III thymomas and thymic carcinomas using the Japanese Association for Research on the Thymus (JART) database; they found that PORT did not improve the RFS or OS of patients with stage II or III thymomas, but did improve the RFS of patients with stage 
Table 3 Univariate and multivariate analysis of recurrence-free survival in patients with stages II and III thymoma

\begin{tabular}{|c|c|c|c|c|c|c|c|c|}
\hline \multirow{2}{*}{ Variables } & \multicolumn{4}{|c|}{ Stage II $(n=346)$} & \multicolumn{4}{|c|}{ Stage III ( $n=58)$} \\
\hline & HR (95\% Cl) & $P$ value & $\mathrm{HR}(95 \% \mathrm{Cl})$ & $P$ value & $\mathrm{HR}(95 \% \mathrm{Cl})$ & $P$ value & $\mathrm{HR}(95 \% \mathrm{Cl})$ & $P$ value \\
\hline Age & $1.00(0.96-1.05)$ & 0.893 & 0.99 (0.94-1.05) & 0.742 & $0.98(0.95-1.01)$ & 0.163 & $0.91(0.86-0.97)$ & 0.001 \\
\hline Sex & & 0.485 & & 0.135 & & 0.543 & & 0.316 \\
\hline Female & $0.70(0.25-1.92)$ & & $0.40(0.12-1.33)$ & & $0.73(0.27-1.99)$ & & $0.53(0.16-1.82)$ & \\
\hline MG & $0.62(0.17-2.20)$ & 0.456 & $0.92(0.19-4.47)$ & 0.912 & $1.61(0.60-4.38)$ & 0.347 & $0.63(0.13-3.05)$ & 0.563 \\
\hline Neoadjuvant CT & $12.58(1.62-97.87)$ & 0.016 & $45.84(4.01-524.19)$ & 0.002 & $2.10(0.67-6.63)$ & 0.206 & $3.63(0.59-22.41)$ & 0.164 \\
\hline Total thymectomy & $0.22(0.04-1.19)$ & 0.078 & $0.28(0.06-1.28)$ & 0.100 & $1.48(0.48-4.54)$ & 0.497 & $0.75(0.12-4.80)$ & 0.763 \\
\hline$A, A B, B 1$ & Reference & & Reference & & Reference & & Reference & \\
\hline B2, B3 & $2.42(0.86-6.81)$ & & $1.02(0.26-3.94)$ & & $2.73(0.62-12.00)$ & & $6.76(1.21-37.70)$ & \\
\hline Pathologic size & $1.14(0.99-1.32)$ & 0.075 & $1.25(1.05-1.49)$ & 0.012 & $0.94(0.81-1.10)$ & 0.467 & $0.89(0.77-1.03)$ & 0.105 \\
\hline Ro resection & $0.27(0.08-0.98)$ & 0.046 & $0.09(0.02-0.43)$ & 0.002 & $0.79(0.81-1.10)$ & 0.467 & $0.13(0.02-0.74)$ & 0.021 \\
\hline POCT & $6.69(2.07-21.60)$ & 0.001 & $2.65(0.49-14.16)$ & 0.256 & $1.37(0.28-6.59)$ & 0.699 & $1.37(0.33-5.75)$ & 0.664 \\
\hline PORT & $0.78(0.28-2.14)$ & 0.623 & $1.01(0.31-3.33)$ & 0.987 & $0.35(0.12-1.00)$ & 0.049 & $0.10(0.02-0.50)$ & 0.005 \\
\hline
\end{tabular}

$\mathrm{HR}$, hazard ratio; Cl, confidence interval; MG, myasthenia gravis; CT, chemotherapy; WHO, World Health Organization; R0 resection, complete resection; POCT, postoperative chemotherapy; PORT, postoperative radiotherapy.

II and III thymic carcinomas (15). In contrast, Jackson et al. used the National Cancer Database in the United States to investigate the impact of PORT on 4,056 patients with stage II and III thymoma and thymic carcinoma, and found PORT to be associated with superior OS after performing PSM. Subset analysis revealed longer OS rates in patients with stage IIB-III disease as well as in those with positive margins (26).

Thymic carcinoma is distinct from thymoma, as the former has more aggressive features and is associated with a low survival rate $(15,23,27)$. Our study evaluated the efficacy of PORT in patients with stages II and III thymoma but did not include those with thymic carcinoma. PSM was performed to minimize bias caused by possible confounding factors, and subgroup analyses according to the M-K stage were also performed owing to its being the most important prognostic factor (15). While previous studies have analyzed the efficacy of PORT in patients with completely resected tumors, we included those with both complete and incomplete resections as we posited that PORT could be beneficial in both situations (28).

In Korea, the choice of PORT for thymoma patients is dependent on separate institutional guidelines or individual physicians. PORT was more commonly administered to patients in the KART database $(63.6 \%$ and $76.6 \%$ of stage II and stage III patients, respectively) than to those in the JART database $(23.8 \%$ and $45.6 \%$ of stage II and stage III patients, respectively) (15). However, there was no precise indication for postoperative irradiation in the KART database, and the reason PORT was selected for each patient was unclear since decisions varied by institution and individual physician. However, such heterogeneity in patients receiving PORT in our study may lower the selection bias and further lend credence to the positive impact of PORT.

In a study of the ITMIG database, Huang et al. found that the median OS of patients with thymoma was 18.9 years, which is relatively longer than that in patients with other solid tumors (27). Given the long OS, it is important to investigate RFS, which may be a more pertinent prognostic 
Table 4 Univariate and multivariate analysis of overall survival in patients with stages II and III thymoma

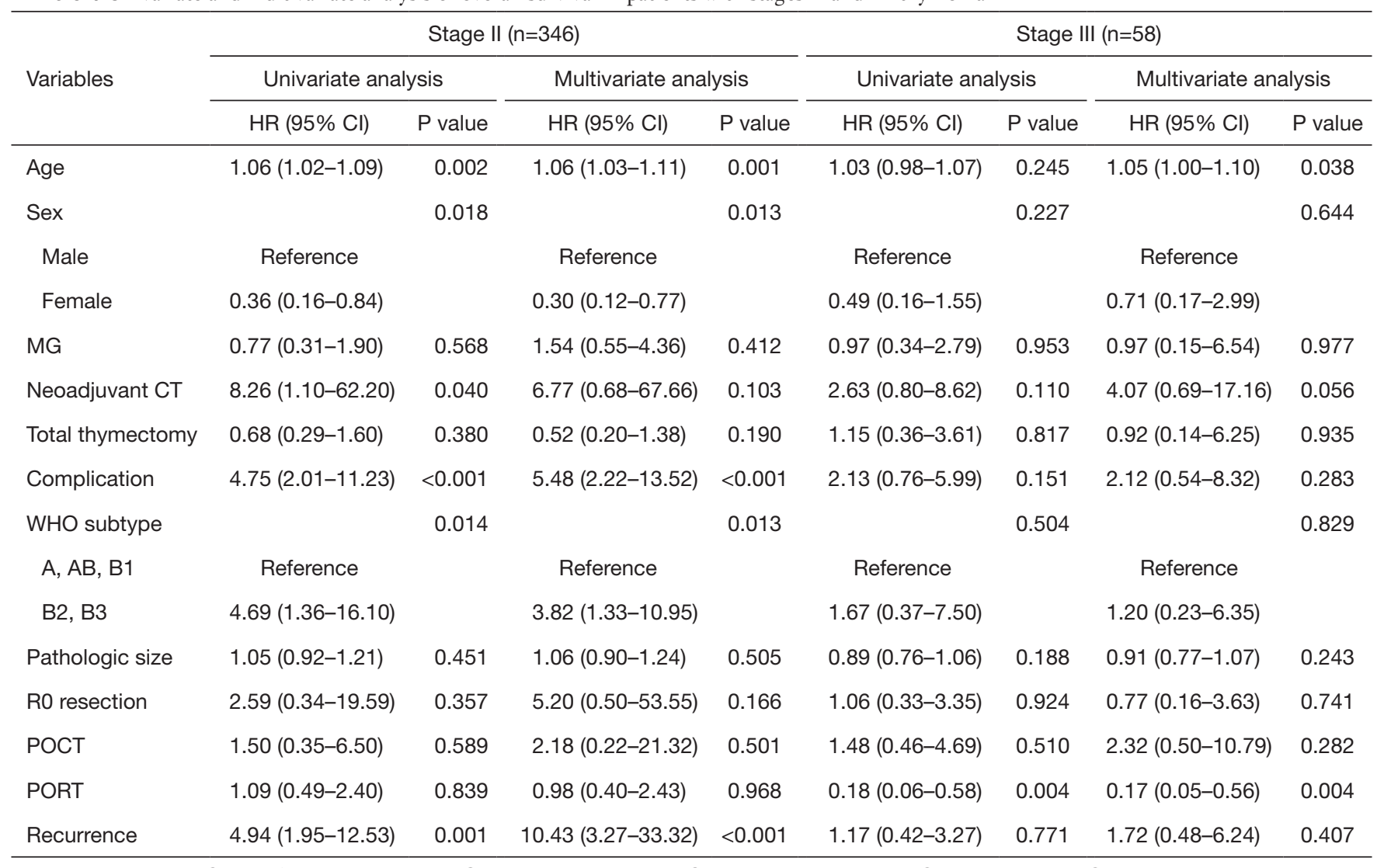

$\mathrm{HR}$, hazard ratio; $\mathrm{Cl}$, confidence interval; MG, myasthenia gravis; CT, chemotherapy; WHO, World Health Organization; RO resection, complete resection; POCT, postoperative chemotherapy; PORT, postoperative radiotherapy.

factor. In our study, we investigated both RFS and OS as determinants of prognosis in thymoma patients. While PORT produced no differences in the RFS and OS rates of the patients overall or only in those with stage II thymomas, it was found to significantly improve both RFS and OS in patients with stage III disease. It was difficult to explain why PORT improved the survival of thymoma only in stage III. However, stage III thymoma has a greater risk than stage II, thus, PORT could be helpful to patients with stage III thymoma. Nonetheless, there should be further investigation, as this is contested topic. Meanwhile, the WHO subtype analysis was not considered in the study design. However, additional analysis was performed using the same database. PORT had a positive impact on stage III and WHO B2/B3 thymoma ( $\mathrm{P}=0.056$ for RFS and $\mathrm{P}=0.002$ for OS; $\mathrm{n}=41$ ), but not on stage II and WHO B2/B3 thymoma ( $\mathrm{P}=0.919$ for RFS and $\mathrm{P}=0.228$ for $\mathrm{OS} ; \mathrm{n}=127$ ). However, further evaluation is warranted.

Generally, patients who experienced disease recurrence or who underwent PORT visited the hospital more often, and had longer follow-up periods than those who did not experience recurrences. Therefore, the positive impact of PORT could be overestimated, and our results should be interpreted with caution. However, our multivariate analysis results do support our finding of a favorable impact of PORT in patients with stage III thymomas.

There currently is no consensus on whether PORT has a positive impact on OS or RFS. In this study, the benefit of PORT on OS and RFS in stage III thymoma was shown. However, the study group was relatively small. Therefore, in conclusion, it appears that PORT could improve the RFS and OS in stage III thymoma.

\section{Limitations}

This study had some key limitations. First, the KART database we used was assembled from multiple institutions and was analyzed retrospectively. Second, the data collection 
period spanned longer than a decade, and some older records lacked certain information such as the indication for neoadjuvant therapy. Third, the KART database is not based on a nationwide pool of patients, and patients may have been lost to follow-up if they subsequently visited other institutions; this may have led to the overor underestimation of the follow-up period. Despite these limitations, however, the study has several strengths. It comprised a large population of thymoma patients listed in a multi-institutional database, which improved the pool's heterogeneity. PSM also adjusted for possible confounding factors between groups, which improved the objectivity of our results.

\section{Conclusions}

PORT can significantly improve the RFS and OS rates of patients with stage III thymoma; however, PORT does not appear to impact the survival of patients with stage II disease.

\section{Acknowledgments}

Funding: None.

\section{Footnote}

Reporting Checklist: The authors have completed the STROBE reporting checklist. Available at http://dx.doi. org/10.21037/jtd-20-1713

Peer Review File: Available at http://dx.doi.org/10.21037/jtd20-1713

Conflicts of Interest: All authors have completed the ICMJE uniform disclosure form (available at http://dx.doi. org/10.21037/jtd-20-1713). The authors have no conflicts of interest to declare.

Ethical Statement: The authors are accountable for all aspects of the work in ensuring that questions related to the accuracy or integrity of any part of the work are appropriately investigated and resolved. The study was conducted in accordance with the Declaration of Helsinki (as revised in 2013). The study was approved by institutional review board (No. 4-2020-0861) and informed consent was taken from all individual participants.
Open Access Statement: This is an Open Access article distributed in accordance with the Creative Commons Attribution-NonCommercial-NoDerivs 4.0 International License (CC BY-NC-ND 4.0), which permits the noncommercial replication and distribution of the article with the strict proviso that no changes or edits are made and the original work is properly cited (including links to both the formal publication through the relevant DOI and the license). See: https://creativecommons.org/licenses/by-nc-nd/4.0/.

\section{References}

1. Gadalla SM, Rajan A, Pfeiffer R, et al. A population-based assessment of mortality and morbidity patterns among patients with thymoma. Int J Cancer 2011;128:2688-94.

2. Duwe BV, Sterman DH, Musani AI. Tumors of the mediastinum. Chest 2005;128:2893-909.

3. Chen G, Marx A, Chen WH, et al. New WHO histologic classification predicts prognosis of thymic epithelial tumors. Cancer 2002;95:420-9.

4. Masaoka A, Monden Y, Nakahara K, et al. Follow-up study of thymomas with special reference to their clinical stages. Cancer 1981;48:2485-92.

5. Regnard JF, Magdeleinat P, Dromer C, et al. Prognostic factors and long-term results after thymoma resection: a series of 307 patients. J Thorac Cardiovasc Surg 1996;112:376-84.

6. Masaoka A. Staging system of thymoma. J Thorac Oncol 2010;5:S304-12.

7. Koga K, Matsuno Y, Noguchi M, et al. A review of 79 thymomas: modification of staging system and reappraisal of conventional division into invasive and non-invasive thymoma. Pathol Int 1994;44:359-67.

8. Onuki T, Ishikawa S, Yamamoto T, et al. Pathologic radioresponse of preoperatively irradiated invasive thymomas. J Thorac Oncol 2008;3:270-6.

9. Gomez D, Komaki R, Yu J, et al. Radiation therapy definitions and reporting guidelines for thymic malignancies. J Thorac Oncol 2011;6:S1743-8.

10. Mangi AA, Wright CD, Allan JS, et al. Adjuvant radiation therapy for stage II thymoma. Ann Thorac Surg 2002;74:1033-7.

11. Mangi AA, Wain JC, Donahue DM, et al. Adjuvant radiation of stage III thymoma: Is it necessary? Ann Thorac Surg 2005;79:1834-9.

12. Korst RJ, Kansler AL, Christos PJ, et al. Adjuvant radiotherapy for thymic epithelial tumors: A 
systematic review and meta-analysis. Ann Thorac Surg 2009;87:1641-7.

13. Detterbeck FC, Asamura H, Crowley J, et al. The IASLC/ ITMIG thymic malignancies staging project: development of a stage classification for thymic malignancies. J Thorac Oncol 2013;8:1467-73.

14. Ruffini E, Detterbeck F, Van Raemdonck D, et al. Thymic carcinoma: a cohort study of patients from the European society of thoracic surgeons database. J Thorac Oncol 2014;9:541-8.

15. Omasa M, Date H, Sozu T, et al. Postoperative radiotherapy is effective for thymic carcinoma but not for thymoma in stage II and III thymic epithelial tumors: The Japanese association for research on the thymus database study. Cancer 2015;121:1008-16.

16. Detterbeck FC. Evaluation and treatment of stage I and II thymoma. J Thorac Oncol 2010;5:S318-22.

17. Falkson CB, Bezjak A, Darling G, et al. The management of thymoma: a systemic review and practice guideline. J Thorac Oncol 2009;4:911-9.

18. Yan J, Liu Q, Moseley JN, et al. Adjuvant radiotherapy for stage II and III resected thymoma: A single-institutional experience. Am J Clin Oncol 2016;39:223-7.

19. Hejna M, Haberl I, Raderer M. Nonsurgical management of malignant thymoma. Cancer 1999;85:1871-84.

20. Thomas CR, Wright CD, Loehrer PJ. Thymoma: State of the art. J Clin Oncol 1999;17:2280-9.

21. Forquer JA, Rong N, Fakiris AJ, et al. Postoperative radiotherapy after surgical resection of thymoma: Differing roles in localized and regional disease. Int J Radiat Oncol Biol Phys 2010;76:440-5.

22. Patel S, Macdonald OK, Nagda S, et al. Evaluation of the role of radiation therapy in the management of malignant thymoma. Int J Radiat Oncol Biol Phys 2012;82:1797-801.

23. Kondo K, Monden Y. Therapy for thymic epithelial tumors: a clinical study of 1,320 patients from Japan. Ann Thorac Surg 2003;76:878-84.

24. Rimner A, Yao X, Huang J, et al. Postoperative radiation therapy is associated with longer overall survival in completely resected stage II and III thymoma-an analysis of the International Thymic Malignancies Interest Group retrospective database. J Thorac Oncol 2016;11:1785-92.

25. Lim YJ, Kim E, Kim HJ, et al. Survival impact of adjuvant radiation therapy in Masaoka stage II to IV thymomas: A systematic review and meta-analysis. Int J Radiat Oncol Biol Phys 2016;94:1129-36.

26. Jackson MW, Palma DA, Camidge DR, et al. The impact of postoperative radiotherapy for thymoma and thymic carcinoma. J Thorac Oncol 2017;12:734-44.

27. Huang J, Ahmad U, Antonicelli A, et al. Development of the international thymic malignancy interest group international database: an unprecedented resource for the study of a rare group of tumors. J Thorac Oncol 2014;9:1573-8.

28. Curran WJ, Kornstein MJ, Brooks JJ, et al. Invasive thymoma: the role of mediastinal irradiation following complete or incomplete surgical resection. J Clin Oncol 1988;6:1722-7.
Cite this article as: Song SH, Suh JW, Yu WS, Byun GE, Park SY, Lee CY, Kim DJ, Paik HC, Chung KY, Lee GD, Choi S, Kim HR, Kim YH, Kim DK, Park SI, Cho JH, Kim HK, Choi YS, Kim J, Zo JI, Shim YM, Hwang Y, Park S, Park IK, Kang $\mathrm{CH}$, Kim YT, Lee JG. The role of postoperative radiotherapy in stage II and III thymoma: a Korean multicenter database study. J Thorac Dis 2020;12(11):6680-6689. doi: 10.21037/jtd-201713 